\title{
A White Noise Approach to Stochastic Differential Equations Driven by Wiener and Poisson Processes
}

\author{
H. Holden, B. ØKSENDAL
}

\section{Introduction}

The main idea behind the white noise approach to stochastic partial differential equations (SPDEs) can be described as follows:

Recall the fact that even quite simple SPDEs, like the stochastic Poisson equation

$$
\begin{aligned}
\Delta F(x) & =-W(x) ; \quad x \in D \subset \mathbb{R}^{d}, \quad d>3 \\
F(x) & =0 ; \quad x \in \partial D
\end{aligned}
$$

(where $\Delta=\sum_{i=1}^{d} \partial^{2} / \partial x_{i}^{2}$ is the Laplacian and $W$ is Gaussian white noise), do not allow solutions which are ordinary stochastic processes/fields $F(x, \omega): \bar{D} \times \Omega \rightarrow \mathbb{R}$. However, Walsh [9] proved that solutions exist in the distribution sense:

For almost all $\omega$ the map $x \mapsto F(x, \omega)$ is a distribution in $x$.

This approach works fine for linear equations like (1.1), but requires more work in the nonlinear case. It is, however, possible also to handle some such cases by introducing Colombeau random generalized functions. See [7] and the references therein.

The white noise approach looks for generalized solutions in the following sense:

$$
\text { For all } x \text { the map } \omega \mapsto F(x, \omega) \text { is a stochastic distribution in } \omega \text {. }
$$

Since there is a natural product in the stochastic distribution spaces (the Wick product), this approach has the advantage of extending easily to many nonlinear SPDEs, provided they are interpreted in the Wick sense. Moreover, there is an 
efficient solution algorithm for such equations, based on the Hermite transform (see, e.g., [6, Theorem 4.1.1], and Section 3 in this paper).

The theory of Gaussian white noise and its applications to stochastic differential equations (SDEs), both ordinary and partial, are now well-known. See for example the presentations in [5] and [6]. It is also known-although not so widely-that a similar type of theory exists for Poissonian white noise. Moreover, this Poissonian white noise theory has similar applications to SDEs driven by the Poisson process. In fact, as described in Section 2 there is even a unitary correspondence $\mathcal{U}$ between the solutions of Gaussian and Poissonian white noise equations, provided they both are of Wick type. See the exposition in [1] and the references therein. A brief survey of this connection can be found in [6, Section 4.9].

However, this connection does not immediately explain how the white noise theory can be modified to cover SDEs driven by combinations of Gaussian and Poissonian white noise. The purpose of this paper is to outline how this can be done and at the same time review the separate Gaussian and Poissonian cases and the relation between them in more detail.

In Section 2 we briefly review the white noise theory in the Gaussian and Poissonian cases separately and some relations between them.

Then in Section 3 we outline how to combine the two white noise theories into one theory that cover equations driven by both Gaussian and Poissonian noise.

In Section 4 we illustrate the theory by considering some examples.

\section{A Review of the Gaussian and Poissonian White Noise Theories - and Relations between Them}

Let $\mathcal{S}\left(\mathbb{R}^{d}\right)$ denote the Schwartz space of rapidly decreasing smooth functions on $\mathbb{R}^{d}$ and let $\mathcal{S}^{\prime}\left(\mathbb{R}^{d}\right)$ be its dual (the space of tempered distributions). Then by the Bochner-Minlos theorem there exists a unique probability measure $\mu$ on $\Omega_{1}:=\mathcal{S}^{\prime}\left(\mathbb{R}^{d}\right)$ defined by the property that

$$
\int_{\Omega_{1}} e^{i\langle\omega, \phi\rangle} d \mu\left(\omega_{1}\right)=\exp \left(-\frac{1}{2}\|\phi\|^{2}\right)
$$

for all $\phi \in \mathcal{S}\left(\mathbb{R}^{d}\right)$, where $\|\phi\|^{2}=\int_{\mathbb{R}^{d}} \phi(x)^{2} d x$ and $\left\langle\omega_{1}, \phi\right\rangle$ is the action of $\omega_{1} \in \Omega_{1}$ on $\phi \in \mathcal{S}\left(\mathbb{R}^{d}\right)$. (See, e.g., [6, Section 2.1] for more details.) The measure $\mu$ is called the 
Gaussian white noise probability measure.

Similarly, again by the Bochner-Minlos theorem, there exists a unique probability measure $\nu$ on $\Omega_{2}:=\mathcal{S}^{\prime}\left(\mathbb{R}^{d}\right)$ defined by the property that

$$
\int_{\Omega_{2}} e^{i\left\langle\omega_{2}, \phi\right\rangle} d \nu\left(\omega_{2}\right)=\exp \left(\int_{\mathbb{R}^{d}}\left(e^{i \phi(x)}-1\right) d x\right)
$$

for all $\phi \in \mathcal{S}\left(\mathbb{R}^{d}\right)$, where (again) $\left\langle\omega_{2}, \phi\right\rangle$ is the action of $\omega_{2} \in \Omega_{2}$ on $\phi \in \mathcal{S}\left(\mathbb{R}^{d}\right)$. The measure $\nu$ is called the Poissonian white noise probability measure.

In the Gaussian case one exploits the isometry

$$
E_{\mu}\left[\langle\cdot, \phi\rangle^{2}\right]=\|\phi\|^{2} ; \quad \phi \in S\left(\mathbb{R}^{d}\right)
$$

to define

$$
\left\langle\omega_{1}, \psi\right\rangle=\lim _{n \rightarrow \infty}\left\langle\omega_{1}, \phi_{n}\right\rangle
$$

with limit in $L^{2}(\mu)$, for all $\psi \in L^{2}\left(\mathbb{R}^{d}\right)$, where $\phi_{n}$ is a sequence in $\mathcal{S}\left(\mathbb{R}^{d}\right)$ such that $\phi_{n} \rightarrow \psi$ in $L^{2}\left(\mathbb{R}^{d}\right)$. In particular, this defines $d$-parameter Brownian motion as (the $x$-continuous version of)

$$
B\left(x, \omega_{1}\right):=\left\langle\omega_{1}, \mathcal{X}_{[0, x]}\right\rangle ; \quad x \in \mathbb{R}^{d}, \omega_{1} \in \Omega_{1}
$$

where

$$
\mathcal{X}_{[0, x]}\left(t_{1}, \ldots, t_{d}\right)=\mathcal{X}_{\left[0, x_{1}\right]}\left(t_{1}\right) \cdots \mathcal{X}_{\left[0, x_{d}\right]}\left(t_{d}\right)
$$

if $x=\left(x_{1}, \ldots, x_{d}\right)$ with $x_{j} \geq 0$ for $j=1, \ldots, d$. (If $x_{j}<0$, we put $\mathcal{X}_{\left[0, x_{j}\right]}=$ $\left.-\mathcal{X}_{\left[-x_{j}, 0\right]} \cdot\right)$

Similarly, in the Poissonian case one exploits the isometry

$$
E_{\nu}\left[\left(\langle\cdot, \phi\rangle-\int_{\mathbb{R}^{d}} \phi(x) d x\right)^{2}\right]=\|\phi\|^{2} ; \quad \phi \in \mathcal{S}\left(\mathbb{R}^{d}\right)
$$

to define

$$
\left\langle\omega_{2}, \psi\right\rangle=\lim _{n \rightarrow \infty}\left\langle\omega_{2}, \phi_{n}\right\rangle
$$

with limit in $\left.L^{1}(\nu) \cap L^{2}(\nu)\right)$, for all $\psi \in L^{2}(\nu) \cap L^{2}(\nu)$, where $\phi_{n}$ is a sequence in $\mathcal{S}\left(\mathbb{R}^{d}\right)$ converging to $\psi$ in $L^{1}(\nu) \cap L^{2}(\nu)$. In particular, this defines the $d$-parameter Poisson process as (the right-continuous version of)

$$
P\left(x, \omega_{2}\right):=\left\langle\omega_{2}, \mathcal{X}_{[0, x]}\right\rangle ; \quad x \in \mathbb{R}^{d}, \omega_{2} \in \Omega_{2} .
$$


The compensated Poission process is then defined by

$$
Q\left(x, \omega_{2}\right):=P\left(x, \omega_{2}\right)-\prod_{j=1}^{d} x_{j} ; \quad x=\left(x_{1}, \ldots, x_{d}\right) .
$$

From here on one can construct stochastic integrals with respect to $B$ and $Q$, respectively and the corresponding stochastic polynomials and so on. We summarize the corresponding concepts in the two cases in the list below. Unless otherwise mentioned we use the terminology of [6]. In particular, recall that $\left\{\eta_{k}\right\}_{k=1}^{\infty}$ is the basis of $L^{2}\left(\mathbb{R}^{d}\right)$ consisting of tensor products of the Hermite functions

$$
\xi_{n}(t)=\pi^{-1 / 4}((n-1) !)^{-1 / 2} e^{-x^{2} / 2} h_{n-1}(\sqrt{2} t) ; \quad n \in \mathbb{N} ; \quad t \in \mathbb{R},
$$

where

$$
h_{m}(y)=(-1)^{m} e^{y^{2} / 2} \frac{d^{m}}{d y^{m}}\left(e^{-y^{2} / 2}\right) ; \quad m \in \mathbb{N} \cup\{0\} ; \quad y \in \mathbb{R}
$$

are the Hermite polynomials. The symbol $\mathcal{J}$ denotes the set of all multi-indices $\alpha=\left(\alpha_{1}, \ldots, \alpha_{n}\right)$ where the $\alpha_{i}$ 's are nonnegative integers and $n \in \mathbb{N}$.

In the following list we use the following notation. $x=\left(x_{1}, \ldots, x_{d}\right) \in \mathbb{R}^{d}, \alpha=$ $\left(\alpha_{1}, \ldots, \alpha_{n}\right) \in \mathcal{J}, \varepsilon^{(k)}$ denotes the $k$ th unit multi-index, namely $\varepsilon^{(k)}=(0,0, \ldots, 1)$ with 1 on $k$ th place. (In [6] $\varepsilon^{(k)}$ is denoted by $\varepsilon^{k}$.) Furthermore, for any function $\xi(x)$ we define $\bar{\xi}=\int_{\mathbb{R}} \xi(x) d x$. Finally, $\hat{\otimes}$ denotes the symmetric tensor product and $\widehat{L}^{2}\left(\mathbb{R}^{m}\right)$ denotes the symmetric functions in $\widehat{L}^{2}\left(\mathbb{R}^{m}\right)$.

(2.1) White noise probability measure

GAUSSiAn: $\int_{\mathcal{S}^{\prime}\left(\mathbb{R}^{d}\right)} e^{i\left(\omega_{1}, \phi\right\rangle} d \mu\left(\omega_{1}\right)=\exp \left(-\frac{1}{2}\|\phi\|^{2}\right) ; \phi \in \mathcal{S}\left(\mathbb{R}^{d}\right)$.

PoISSONIAN: $\int_{\mathcal{S}^{\prime}\left(\mathbb{R}^{d}\right)} e^{i\left\langle\omega_{2}, \phi\right\rangle} d \nu\left(\omega_{2}\right)=\exp \left(\int_{\mathbb{R}^{d}}\left(e^{i \phi(x)}-1\right) d x\right) ; \phi \in \mathcal{S}\left(\mathbb{R}^{d}\right)$.

(2.2) Processes ${ }^{1}$

Gaussian: Brownian motion $B: B\left(x, \omega_{1}\right)=\left\langle\omega_{1}, \mathcal{X}_{[0, x]}\right\rangle$.

Poissonian: Poisson process $P: P\left(x, \omega_{2}\right)=\left\langle\omega_{2}, \mathcal{X}_{[0, x]}\right\rangle$. Compensated Poisson process $Q: Q\left(x, \omega_{2}\right)=P\left(x, \omega_{2}\right)-\prod_{k=1}^{d} x_{k}$.

\section{(2.3) Stochastic integrals}

\footnotetext{
${ }^{1}$ Note that both $\left\langle\omega_{1}, \mathcal{X}_{[0, x]}\right\rangle$ and $\left\langle\omega_{2}, \mathcal{X}_{[0, x]}\right\rangle$ are only defined almost everywhere with respect to $\mu$ and $\nu$, respectively. Since $\mu$ and $\nu$ are not absolutely continuous with respect to each other, the two processes $B(x)$ and $P(x)$ are not the same even though they are defined by the same expression.
} 
GAUSSIAN: $\left\langle\omega_{1}, \phi\right\rangle=\int_{\mathbb{R}^{d}} \phi(x) d B(x)$ a.s. $\mu$ for all $\phi \in L^{2}\left(\mathbb{R}^{d}\right)$.

POISSONIAN: $\left\langle\omega_{2}, \psi\right\rangle=\int_{\mathbb{R}^{d}} \psi(x) d P(x)$ a.s. $\nu$, and

$\int_{\mathbb{R}^{d}} \psi(x) d Q(x)=\int_{\mathbb{R}^{d}} \psi(x) d P(x)-\bar{\psi}$ for all $\psi \in L^{1}\left(\mathbb{R}^{d}\right) \cap L^{2}\left(\mathbb{R}^{d}\right)$.

\section{(2.4) Stochastic polynomials}

GAUSSIAN: Hermite polynomials $H_{\alpha}\left(\omega_{1}\right)=\prod_{j} h_{\alpha_{j}}\left(\left\langle\omega_{1}, \eta_{j}\right\rangle\right)$,

Poissonian: Charlier polynomials $C_{\alpha}\left(\omega_{2}\right)=C_{|\alpha|}(\omega_{2} ; \overbrace{\eta_{1}, \ldots, \eta_{1}}^{\alpha_{1}}, \ldots, \overbrace{\eta_{m}, \ldots, \eta_{m}}^{\alpha_{m}})$, where

$$
\begin{aligned}
& C_{n}\left(\omega_{2} ; \psi_{1}, \ldots, \psi_{n}\right) \\
& \quad=\left.\frac{\partial^{n}}{\partial u_{1} \cdots \partial u_{n}} \exp \left(\left\langle\omega_{2}, \ln \left(1+\sum_{j=1}^{n} u_{j} \psi_{j}\right)\right\rangle-\sum_{j=1}^{n} u_{j} \int_{\mathbb{R}^{d}} \psi_{j}(x) d x\right)\right|_{u_{1}=\cdots=u_{n}=0},
\end{aligned}
$$

for $\psi_{j} \in S\left(\mathbb{R}^{d}\right) ; 1 \leq j \leq n$.

\section{(2.4a) Example}

GAUSSIAN: $H_{\varepsilon^{(k)}}\left(\omega_{1}\right)=h_{1}\left(\left\langle\omega_{1}, \eta_{k}\right\rangle\right)=\left\langle\omega_{1}, \eta_{k}\right\rangle=\int_{\mathbb{R}^{d}} \eta_{k}(x) d B(x)$.

Poissonian: $C_{\varepsilon^{(\ell)}}\left(\omega_{2}\right)=\left\langle\omega_{2}, \eta_{\ell}\right\rangle-\bar{\eta}_{\ell}=\int_{\mathbb{R}^{d}} \eta_{\ell}(x) d Q(x)$.

(2.5) Expansion in terms of iterated integrals

GAUSSIAN: $f \in L^{2}(\mu) \Rightarrow f=\sum_{n=0}^{\infty} \int_{\mathbb{R}^{n d}} f_{n}(x) d B^{\otimes n}(x)$, where $f_{n} \in \widehat{L}^{2}\left(\mathbb{R}^{n d}\right)$.

$\|f\|_{L^{2}(\mu)}^{2}=\sum_{n=0}^{\infty} n !\left\|f_{n}\right\|_{L^{2}\left(\mathbb{R}^{n d}\right)}^{2}$.

POISSONIAN: $g \in L^{2}(\mu) \Rightarrow g=\sum_{n=0}^{\infty} \int_{\mathbb{R}^{n d}} g_{n}(x) d Q^{\otimes n}(x)$, where $g_{n} \in \widehat{L}^{2}\left(\mathbb{R}^{n d}\right)$.

$\|g\|_{L^{2}(\nu)}^{2}=\sum_{n=0}^{\infty} n !\left\|g_{n}\right\|_{L^{2}\left(\mathbb{R}^{n d}\right)}^{2}$.

\section{(2.6) Expansion in terms of polynomials}

GAUSSIAN: $f \in L^{2}(\mu) \Rightarrow f\left(\omega_{1}\right)=\sum_{\alpha \in \mathcal{J}} a_{\alpha} H_{\alpha}\left(\omega_{1}\right)$, where $a_{\alpha} \in \mathbb{R}$.

$\|f\|_{L^{2}(\mu)}^{2}=\sum_{\alpha \in \mathcal{J}} a_{\alpha}^{2} \alpha$ !.

PoIssonian: $g \in L^{2}(\nu) \Rightarrow g\left(\omega_{2}\right)=\sum_{\beta \in \mathcal{J}} b_{\beta} C_{\beta}\left(\omega_{2}\right)$, where $b_{\beta} \in \mathbb{R}$. $\|g\|_{L^{2}(\nu)}^{2}=\sum_{\beta \in \mathcal{J}} b_{\beta}^{2} \beta$ !.

\section{(2.7) Relation between the two expansions}

GAUSSIAN: $\int_{\mathbb{R}^{|\alpha| d}} \eta^{\widehat{\otimes} \alpha} d B^{\otimes|\alpha|}=H_{\alpha}\left(\omega_{1}\right)$.

POISSONIAN: $\int_{\mathbb{R}^{|\alpha| d}} \eta^{\widehat{\otimes} \alpha} d Q^{\otimes|\alpha|}=C_{\alpha}\left(\omega_{2}\right)$.

(2.8) Kondratiev norms, $-1 \leq \rho \leq 1, q \in \mathbb{Z}$

GAUSSIAN: $F\left(\omega_{1}\right)=\sum_{\alpha \in \mathcal{J}} a_{\alpha} H_{\alpha}\left(\omega_{1}\right) \Rightarrow\|F\|_{\rho, q, \mu}^{2}=\sum_{\alpha} a_{\alpha}^{2}(\alpha !)^{1+\rho}(2 \mathbb{N})^{q \alpha}$.

POISSONIAN: $G\left(\omega_{2}\right)=\sum_{\beta \in \mathcal{J}} b_{\beta} C_{\beta}\left(\omega_{2}\right) \Rightarrow\|G\|_{\rho, q, \nu}^{2}=\sum_{\beta} b_{\beta}^{2}(\beta !)^{1+\rho}(2 \mathbb{N})^{q \beta}$. 
(2.9) Kondratiev Hilbert spaces

GAUSSIAN: $(\mathcal{S})_{\rho, q, \mu}:=\left\{F ;\|F\|_{\rho, q, \mu}<\infty\right\}$.

POISSONIAN: $(\mathcal{S})_{\rho, q, \nu}:=\left\{G ;\|G\|_{\rho, q, \nu}<\infty\right\}$.

(2.10) Kondratiev stochastic test function spaces, $0 \leq \rho \leq 1$

GAUSSIAN: $(\mathcal{S})_{\rho, \mu}=\bigcap_{r \in \mathbb{N}}(\mathcal{S})_{\rho, r, \mu}$, with projective topology.

PoISSONIAN: $(\mathcal{S})_{\rho, \nu}=\bigcap_{r \in \mathbb{N}}(\mathcal{S})_{\rho, r, \nu}$, with projective topology.

(2.11) Kondratiev stochastic distribution spaces, $0 \leq \rho \leq 1$

GaUssian: $(\mathcal{S})_{-\rho, \mu}=\bigcup_{r \in \mathbb{N}}(\mathcal{S})_{-\rho,-r, \mu}$, with inductive topology.

PoISSONIAN: $(\mathcal{S})_{-\rho, \nu}=\bigcup_{r \in \mathbb{N}}(\mathcal{S})_{-\rho,-r, \nu}$, with inductive topology.

(2.12) Hida spaces

GAUSSIAN: $(\mathcal{S})_{\mu}:=(\mathcal{S})_{0, \mu}, \quad(\mathcal{S})_{\mu}^{*}:=(\mathcal{S})_{-0, \mu}$.

POISSONIAN: $(\mathcal{S})_{\nu}:=(\mathcal{S})_{0, \nu}, \cdots \quad(\mathcal{S})_{\nu}^{*}:=(\mathcal{S})_{-0, \nu}$.

(2.13) White noise

GAUSSIAN: $W\left(x, \omega_{1}\right)=\sum_{k=1}^{\infty} \eta_{k}(x) H_{\varepsilon^{(k)}}\left(\omega_{1}\right), \quad W(x, \cdot) \in(\mathcal{S})_{\mu}^{*}$ for all $x$.

PoIssonian: $V\left(x, \omega_{2}\right)=\sum_{k=1}^{\infty} \eta_{k}(x) C_{\varepsilon^{(k)}}\left(\omega_{2}\right), \quad V(x, \cdot) \in(\mathcal{S})_{\nu}^{*}$ for all $x$.

(2.14) Wick product

GAUSSIAN: $\left(\sum_{\alpha} a_{\alpha} H_{\alpha}\right) \diamond\left(\sum_{\beta} b_{\beta} H_{\beta}\right)=\sum_{\alpha, \beta} a_{\alpha} b_{\beta} H_{\alpha+\beta}=\sum_{\gamma}\left(\sum_{\alpha+\beta=\gamma} a_{\alpha} b_{\beta}\right) H_{\gamma}$.

Poissonian: $\left(\sum_{\alpha} a_{\alpha} C_{\alpha}\right) \diamond\left(\sum_{\beta} b_{\beta} C_{\beta}\right)=\sum_{\alpha, \beta} a_{\alpha} b_{\beta} C_{\alpha+\beta}=\sum_{\gamma}\left(\sum_{\alpha+\beta=\gamma} a_{\alpha} b_{\beta}\right) C_{\gamma}$.

(2.15) Properties of the Wick product

Gaussian: $f, g \in(\mathcal{S})_{1, \mu} \Rightarrow f \diamond g \in(\mathcal{S})_{1, \mu}, \quad F, G \in(\mathcal{S})_{-1, \mu} \Rightarrow F \diamond G \in(\mathcal{S})_{-1, \mu}$.

Poissonian: $f, g \in(\mathcal{S})_{1, \nu} \Rightarrow f \diamond g \in(\mathcal{S})_{1, \nu}, \quad F, G \in(\mathcal{S})_{-1, \nu} \Rightarrow F \diamond G \in(\mathcal{S})_{-1, \nu}$.

(2.16) Wick products in terms of iterated integrals

GAUSSIAN: $\left(\int_{\mathbb{R}^{n d}} f_{n} d B^{\otimes n}\right) \diamond\left(\int_{\mathbb{R}^{m d}} f_{m} d B^{\otimes m}\right)=\int_{\mathbb{R}^{(n+m) d}}\left(f_{n} \widehat{\otimes} f_{m}\right) d B^{\otimes(n+m)}$.

POISSONIAN: $\left(\int_{\mathbb{R}^{n d}} g_{n} d Q^{\otimes n}\right) \diamond\left(\int_{\mathbb{R}^{m d}} g_{m} d Q^{\otimes m}\right)=\int_{\mathbb{R}^{(n+m) d}}\left(g_{n} \widehat{\otimes} g_{m}\right) d Q^{\otimes(n+m)}$.

(2.17) Wick powers and iterated integrals ${ }^{2}$

GAUSSIAN: $\left\langle\omega_{1}, \phi\right\rangle^{\diamond n}=\int_{\mathbb{R}^{n d}} \phi^{\otimes n} d B^{\otimes n}$ for $\phi \in L^{2}\left(\mathbb{R}^{d}\right)$.

POISSONIAN: $\left(\left\langle\omega_{2}, \psi\right\rangle-\bar{\psi}\right)^{\diamond n}=\int_{\mathbb{R}^{n d}} \psi^{\otimes n} d Q^{\otimes n}$ for $\psi \in L^{1}\left(\mathbb{R}^{d}\right) \cap L^{2}\left(\mathbb{R}^{d}\right)$.

(2.18) Wick exponentials ${ }^{3}$

\footnotetext{
${ }^{2}$ These formulas follow by combining (2.3) and (2.16).

${ }^{3}$ The Gaussian case can be found, e.g., in [6, Lemma 2.6.16]. For a proof in the Poissonian case and when $\alpha=1$ see Corollary 2.3 below.
} 
GAUSSIAN: $\exp ^{\diamond}\left(\left\langle\omega_{1}, \phi\right\rangle\right):=\sum_{n=0}^{\infty} \frac{1}{n !}\left\langle\omega_{1}, \phi\right\rangle^{\diamond n}=\exp \left(\left\langle\omega_{1}, \phi\right\rangle-\frac{1}{2}\|\phi\|^{2}\right)$

for all $\phi \in L^{2}\left(\mathbb{R}^{d}\right)$.

POISSONIAN: $\exp ^{\diamond}\left(\left\langle\omega_{2}, \psi\right\rangle\right):=\sum_{n=0}^{\infty} \frac{1}{n !}\left\langle\omega_{2}, \psi\right\rangle^{\diamond n}=\exp \left(\left\langle\omega_{2}, \ln (1+\psi)\right\rangle\right)$

for all $\psi \in L^{1}\left(\mathbb{R}^{d}\right) \cap L^{2}\left(\mathbb{R}^{d}\right), \psi \geq-1+\varepsilon$ for some $\varepsilon>0$.

(2.19) Wick powers and Wick exponentials ${ }^{4}$

GAUSSIAN: $\left\langle\omega_{1}, \phi\right\rangle^{\diamond n}=\left.\frac{\partial^{n}}{\partial u^{n}} \exp ^{\diamond}\left(\left\langle\omega_{1}, u \phi\right\rangle\right)\right|_{u=0}$

$=\left.\frac{\partial^{n}}{\partial u^{n}} \exp \left(\left\langle\omega_{1}, u \phi\right\rangle-\frac{1}{2} u^{2}\|\phi\|^{2}\right)\right|_{u=0}$ for all $\phi \in L^{2}\left(\mathbb{R}^{d}\right)$.

POISSONIAN: $\left\langle\omega_{2}, \psi\right\rangle^{\diamond n}=\left.\frac{\partial^{n}}{\partial u^{n}} \exp ^{\diamond}\left(\left\langle\omega_{2}, u \psi\right\rangle\right)\right|_{u=0}$

$=\frac{\partial^{n}}{\partial u^{n}} \exp \left(\left.\left\langle\omega_{2}, \ln (1+u \psi\rangle\right)\right|_{u=0}\right.$ for all $\psi \in L^{1}\left(\mathbb{R}^{d}\right) \cap L^{2}\left(\mathbb{R}^{d}\right)$.

(2.20) Wick polynomials and Wick exponentials ${ }^{4}$

GAUSSIAN: $\left\langle\omega_{1}, \phi_{1}\right\rangle \diamond \cdots \diamond\left\langle\omega_{1}, \phi_{n}\right\rangle=\left.\frac{\partial^{n}}{\partial u_{1} \cdots \partial u_{n}} \exp ^{\diamond}\left(\left\langle\omega_{1}, \sum_{j=1}^{n} u_{j} \phi_{j}\right\rangle\right)\right|_{u_{1}=\cdots=u_{n}=0}$ $=\left.\frac{\partial^{n}}{\partial u_{1} \cdots \partial u_{n}} \exp \left(\left\langle\omega_{1}, \sum_{j=1}^{n} u_{j} \phi_{j}\right\rangle-\frac{1}{2}\left\|\sum_{j=1}^{n} u_{j} \phi_{j}\right\|^{2}\right)\right|_{u_{1}=\cdots=u_{n}=0}$

for $\phi_{j} \in L^{2}\left(\mathbb{R}^{d}\right)$ for $1 \leq j \leq n$.

POISSONIAN: $\left\langle\omega_{2}, \psi_{1}\right\rangle \diamond \cdots \diamond\left\langle\omega_{2}, \psi_{n}\right\rangle=\left.\frac{\partial^{n}}{\partial u_{1} \cdots \partial u_{n}} \exp ^{\diamond}\left(\left\langle\omega_{2}, \sum_{j=1}^{n} u_{j} \psi_{j}\right\rangle\right)\right|_{u_{1}=\cdots=u_{n}=0}$ $=\left.\frac{\partial^{n}}{\partial u_{1} \cdots \partial u_{n}} \exp \left(\left\langle\omega_{2}, \ln \left(1+\sum_{j=1}^{n} u_{j} \psi_{j}\right)\right\rangle\right)\right|_{u_{1}=\cdots=u_{n}=0}$ for $\psi_{j} \in L^{1}\left(\mathbb{R}^{d}\right) \cap L^{2}\left(\mathbb{R}^{d}\right)$ and $1 \leq j \leq n$.

(2.21) Representation of stochastic polynomials in terms of Wick exponentials ${ }^{5}$

GAUSSIAN: $H_{\alpha}\left(\omega_{1}\right)=\left.\partial^{\alpha} \exp ^{\diamond}\left(\int_{\mathbb{R}^{d}} \sum_{j=1}^{n} u_{j} \eta_{j}(x) d B(x)\right)\right|_{u_{1}=\cdots=u_{n}=0}$,

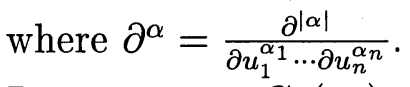

PoISSONIAN: $C_{\alpha}\left(\omega_{2}\right)=\left.\partial^{\alpha} \exp ^{\diamond}\left(\int_{\mathbb{R}^{d}} \sum_{j=1}^{n} u_{j} \eta_{j}(x) d Q(x)\right)\right|_{u_{1}=\cdots=u_{n}=0}$.

\section{(2.22) Skorohod integrals}

GAUSSIAN: $Y\left(t, \omega_{1}\right)=\sum_{n=0}^{\infty} \int_{\mathbb{R}^{n}} f_{n}(t, \cdot) d B^{\otimes n}$, where

$\sum_{n=0}^{\infty}(n+1) !\left\|\widetilde{f}_{n}\right\|_{L^{2}\left(\mathbb{R}^{n+1}\right)}<\infty \Rightarrow \int_{\mathbb{R}} Y(t) \delta B(t):=\sum_{n=0}^{\infty} \int_{\mathbb{R}^{n+1}} \widetilde{f}_{n} d B^{\otimes(n+1)}$. Here $\widetilde{f}_{n}$ is the symmetrization of $f_{n}\left(t, x_{1}, \ldots, x_{n}\right)$ with respect to all its $n+1$ variables.

POISSONIAN: $Z\left(t, \omega_{2}\right)=\sum_{n=0}^{\infty} \int_{\mathbb{R}^{n}} g_{n}(t, \cdot) d Q^{\otimes n}$, where $\sum_{n=0}^{\infty}(n+1) !\left\|\widetilde{g}_{n}\right\|_{L^{2}\left(\mathbb{R}^{n+1}\right)}<\infty \Rightarrow \int_{\mathbb{R}} Z(t) \delta Q(t):=\sum_{n=0}^{\infty} \int_{\mathbb{R}^{n+1}} \widetilde{g}_{n} d Q^{\otimes(n+1)}$.

${ }^{4}$ This follows from (2.18).

${ }^{5}$ The Gaussian case follows from (2.4), the fact that $h_{\alpha_{j}}\left(\left\langle\omega_{1}, \eta_{j}\right\rangle\right)=\left\langle\omega_{1}, \eta_{j}\right\rangle^{\diamond \alpha_{j}}$ (which is seen from (2.7) and (2.17)) plus (2.20). To prove the Poissonian case note that by (2.3) we have, for $\psi \in L^{1}\left(\mathbb{R}^{d}\right) \cap L^{2}\left(\mathbb{R}^{d}\right), \int_{\mathbb{R}^{d}} \psi(x) d Q(x)=\int_{\mathbb{R}^{d}} \psi(x) d P(x)-\bar{\psi}=\left\langle\omega_{2}, \psi\right\rangle-\bar{\psi}$. Therefore, by (2.18) we get $\exp ^{\diamond}\left(\int_{\mathbb{R}^{d}} \psi(x) d Q(x)\right)=\exp ^{\diamond}\left(\left\langle\omega_{2}, \psi\right\rangle-\bar{\psi}\right)=\exp \left(\left\langle\omega_{2}, \ln (1+\psi)\right\rangle-\bar{\psi}\right)$, assuming that $\psi \geq-1+\varepsilon$ for some $\varepsilon>0$. Applying this to appropriate approximations to $\psi_{0}=\sum_{j=1}^{n} u_{j} \eta_{j}$ we see that (2.21) follows from the definition of $C_{\alpha}$ in (2.4). 
(2.23) Connection between Skorohod integrals and Ito-Poisson integrals ${ }^{6}$ GAUSSIAN: $\int_{\mathbb{R}} Y(t) \delta B(t)=\int_{\mathbb{R}} Y(t) d B(t)$ if $Y(t)$ is $\mathcal{F}_{t}$-adapted.

PoIsSONIAN: $\int_{\mathbb{R}} Z(t) \delta Q(t)=\int_{\mathbb{R}} Z(t) d Q(t)$ if $Z(t)$ is adapted to the filtration $\mathcal{G}_{t}$ generated by $\{P(s, \cdot)\}_{s \leq t}$.

(2.24) White noise description of Skorohod integrals ${ }^{6}$

GAUSSIAN: If $Y(t)$ is Skorohod integrable, see (2.22), then

$\int_{\mathbb{R}} Y(t) \delta B(t)=\int_{\mathbb{R}} Y(t) \diamond W(t) d t$.

PoISSONIAN: If $Z(t)$ is Skorohod integrable, see (2.22), then

$\int_{\mathbb{R}} Z(t) \delta Q(t)=\int_{\mathbb{R}} Z(t) \diamond V(t) d t$.

\section{(2.25) Hermite transform}

GAUSSIAN: $F\left(\omega_{1}\right)=\sum_{\alpha \in \mathcal{J}} a_{\alpha} H_{\alpha}\left(\omega_{1}\right) \Rightarrow(\mathcal{H} F)(z)=\sum_{\alpha \in \mathcal{J}} c_{\alpha} z^{\alpha}$

for $z=\left(z_{1}, z_{2}, \ldots\right)$, where $z^{\alpha}=z_{1}^{\alpha_{1}} z_{2}^{\alpha_{2}} \cdots$.

Poissonian: $G\left(\omega_{2}\right)=\sum_{\beta \in \mathcal{J}} b_{\beta} C_{\beta}\left(\omega_{2}\right) \Rightarrow(\mathcal{H} G)(z)=\sum_{\beta \in \mathcal{J}} b_{\beta} z^{\beta}$.

(2.26) $S$-transform (definition), $\rho<1$

GAUSSIAN: $F \in(\mathcal{S})_{-\rho, \mu} \Rightarrow(S F)(\phi)=\left\langle F, \exp ^{\diamond} \int_{\mathbb{R}^{d}} \phi d B\right\rangle$ for all $\phi \in \mathcal{S}\left(\mathbb{R}^{d}\right)$.

POISSONIAN: $G \in(\mathcal{S})_{-\rho, \nu} \Rightarrow(S G)(\phi)=\left\langle G, \exp ^{\diamond} \int_{\mathbb{R}^{d}} \phi d Q\right\rangle$ for all $\phi \in \mathcal{S}\left(\mathbb{R}^{d}\right)$.

(2.27) $S$-transform (properties), $\rho<1$

GAUSSIAN: $F=\sum_{\alpha} a_{\alpha} H_{\alpha}\left(\omega_{1}\right) \in(\mathcal{S})_{-\rho, \mu} \Rightarrow(S F)(\phi)=\sum_{\alpha} a_{\alpha}(\phi, \eta)^{\alpha}$

for all $\phi \in S\left(\mathbb{R}^{d}\right)$.

Poissonian: $G=\sum_{\beta} b_{\beta} C_{\beta}\left(\omega_{2}\right) \in(\mathcal{S})_{-\rho, \nu} \Rightarrow(S G)(\phi)=\sum_{\beta} b_{\beta}(\phi, \eta)^{\beta}$

for all $\phi \in S\left(\mathbb{R}^{d}\right)$.

\section{The unitary correspondence $\mathcal{U}$}

We review some other concepts and results from [1]: Define $\mathcal{U}:(\mathcal{S})_{-1, \mu} \rightarrow(\mathcal{S})_{-1, \nu}$ by

$$
\mathcal{U}\left(\sum_{\alpha} a_{\alpha} H_{\alpha}\right)=\sum_{\alpha} a_{\alpha} C_{\alpha}
$$

Then $\mathcal{U}$ is a continuous bijection and $\left.\mathcal{U}\right|_{(\mathcal{S})_{\rho, q, \mu}}$ is an isometry onto $(\mathcal{S})_{\rho, q, \nu}$ for all $\rho \in[-1,1]$ and all $q \in \mathbb{Z}$. Moreover, $\mathcal{U}$ preserves the Wick product:

$$
\mathcal{U}(F \diamond G)=(\mathcal{U} F) \diamond(\mathcal{U} G)
$$

\footnotetext{
${ }^{6}$ For proofs and more details in the Gaussian case see, e.g., [6, Theorem 2.5.9] and the references therein. For a proof in the Poissonian case, see [6, Theorem 5.1]. This proof is valid in the more general setting of colored noise.
} 
Some examples: We have that

$$
\mathcal{U}\left(\left\langle\omega_{1}, \psi\right\rangle\right)=\left\langle\omega_{2}, \psi\right\rangle-\bar{\psi} ; \quad \psi \in L^{1}\left(\mathbb{R}^{d}\right) \cap L^{2}(\mathbb{R})
$$

which follows from (2.4a). In particular,

$$
\begin{aligned}
& \mathcal{U}\left(B\left(x, \omega_{1}\right)\right)=Q\left(x, \omega_{2}\right), \\
& \mathcal{U}\left(F^{\diamond n}\left(\omega_{1}\right)\right)=(\mathcal{U} F)^{\diamond n}\left(\omega_{2}\right) .
\end{aligned}
$$

by using induction on (2.28). From the Wick-Taylor expansions and (2.31)

$$
\mathcal{U}\left(\exp ^{\diamond} F\left(\omega_{1}\right)\right)=\exp ^{\diamond} \mathcal{U} F\left(\omega_{2}\right)
$$

Recall the following fundamental result from [1]: (For notation see Corollary 4.9.7 in [6].)

Theorem 2.1 Suppose $F(t, x): \mathbb{R}^{d+1} \rightarrow(\mathcal{S})_{-1, \mu}$ is a solution of a Gaussian Wick type stochastic differential equation of the form

$$
A^{\diamond}\left(t, x, \partial_{t}, \nabla_{x}, F, W\right)=0 .
$$

Then $G:=\mathcal{U} F$ is a solution of the corresponding Poissonian Wick type equation

$$
A^{\diamond}\left(t, x, \partial_{t}, \nabla_{x}, G, V\right)=0
$$

and conversely. Here $A^{\diamond}$ denotes the Gaussian (resp. Poissonian) Wick version of a given function $A$.

This elegant result shows that when considering Wick type stochastic differential equations it is - at least in principle- enough to know the solution in the Gaussian case. The solution in the Poissonian case then follows by applying the map $\mathcal{U}$. We illustrate this by considering a simple example:

Example 2.2 Let $X(t)$ be the solution of the (1-parameter) SDE

$$
d X(t)=\psi(t) X(t) d Q(t) ; \quad t>0
$$

where $Q(t)=Q\left(t, \omega_{2}\right)$ is the compensated Poisson process and $\psi(t)$ is a continuous, deterministic function, $\psi(t)>-1+\varepsilon$ for all $t$, for some $\varepsilon>0$. According to the Poissonian white noise theory, the equation can be regarded as a Wick type stochastic differential equation in the (Poisson) Hida space $(\mathcal{S})_{\nu}^{*}$, namely the equation

$$
\frac{d X(t)}{d t}=\psi(t) X(t) \diamond V(t)
$$


where $\diamond$ denotes the Poissonian Wick product and $V(t)=d Q(t) / d t \in(\mathcal{S})_{\nu}^{*}$ is Poissonian white noise.

The corresponding Gaussian white noise differential equation is

$$
\frac{d Y(t)}{d t}=\psi(t) Y(t) \diamond W(t)
$$

where $\diamond$ denotes the Gaussian Wick product and $W(t)=d B(t) / d t \in(\mathcal{S})_{\mu}^{*}$ is Gaussian white noise.

Using Wick calculus on equation (2.35), we get the solution

$$
Y(t)=Y(0) \diamond \exp ^{\diamond}\left(\int_{0}^{t} \psi(s) d B(s)\right)
$$

(see, e.g., [6, Theorem 3.1.2] for a more general calculation).

If $Y(0)=X(0)$ is a constant, then we get

$$
Y(t)=X(0) \exp ^{\diamond}\left(\int_{0}^{t} \psi(s) d B(s)\right)
$$

According to the correspondence $\mathcal{U}$ the solution $X(t)$ of (2.34) is therefore

$$
X(t)=\mathcal{U}(Y(t))=X(0) \exp ^{\diamond}\left(\int_{0}^{t} \psi(s) d Q(s)\right) .
$$

In the Gaussian case we know that

$$
\exp ^{\diamond}\left(\int_{0}^{t} \psi(s) d B(s)\right)=\exp \left(\int_{0}^{t} \psi(s) d B(s)-\frac{1}{2} \int_{0}^{t} \psi(s)^{2} d s\right)
$$

but what is $\exp ^{\diamond}\left(\int_{0}^{t} \psi(s) d Q(s)\right)$ in the Poissonian case? The answer can be found using (2.18):

$$
\begin{aligned}
\exp ^{\diamond}\left(\int_{0}^{t} \psi(s) d Q(s)\right) & =\exp ^{\diamond}\left(\int_{0}^{t} \psi(s) d P(s)-\int_{0}^{t} \psi(s) d s\right) \\
& =\exp ^{\diamond}\left(\left\langle\omega_{2}, \mathcal{X}_{[0, t]} \psi\right\rangle-\int_{0}^{t} \psi(s) d s\right) \\
& =\exp \left(\left\langle\omega_{2}, \ln \left(1+\mathcal{X}_{[0, t]} \psi\right)\right\rangle-\int_{0}^{t} \psi(s) d s\right) \\
& =\exp \left(\int_{0}^{t} \ln (1+\psi(s)) d P(s)-\int_{0}^{t} \psi(s) d s\right)
\end{aligned}
$$

Combining (2.37) and (2.38) we get the following expression for the solution $X(t)$ of (2.33):

$$
X(t)=X(0) \exp \left(\int_{0}^{t} \ln (1+\psi(s)) d P(s)-\int_{0}^{t} \psi(s) d s\right)
$$


It may be instructive to see that we can verify the formula (2.39) by using (2.34) directly as follows:

The Poisson process is described by a sequence of stopping times $0<\tau_{1}<\tau_{2}<\cdots$ at which $P$ jumps with size 1 . Between these stopping times $X(t)$ satisfies the equation

$$
d X(t)=-\psi(t) X(t) d t ; \quad \tau_{k} \leq t<\tau_{k+1},
$$

since $d P(t)=d Q(t)-d t$. Hence

$$
X(t)=X\left(\tau_{k}\right) \exp \left(-\int_{\tau_{k}}^{t} \psi(s) d s\right) ; \quad \tau_{k} \leq t<\tau_{k+1} .
$$

At the jumping times $\tau_{k}$ the value of $X$ increases by

$$
\Delta X\left(\tau_{k}\right):=X\left(\tau_{k}\right)-X\left(\tau_{k}^{-}\right)=\psi\left(\tau_{k}\right) X\left(\tau_{k}^{-}\right)
$$

Hence

$$
X\left(\tau_{k}\right)=X\left(\tau_{k}^{-}\right)\left(1+\psi\left(\tau_{k}\right)\right)=X\left(\tau_{k}^{-}\right) \exp \left(\ln \left(1+\psi\left(\tau_{k}\right)\right)\right)
$$

Combining (2.40) and (2.41) we get

$$
\begin{aligned}
X(t) & =X(0) \prod_{\tau_{k} \leq t} \exp \left(\ln \left(1+\psi\left(\tau_{k}\right)\right)\right) \exp \left(-\int_{\tau_{k}}^{t \wedge \tau_{k+1}} \psi(s) d s\right) \\
& =X(0) \exp \left(\sum_{\tau_{k} \leq t} \ln \left(1+\psi\left(\tau_{k}\right)\right)-\int_{\tau_{k}}^{t \wedge \tau_{k+1}} \psi(s) d s\right) \\
& =X(0) \exp \left(\int_{0}^{t} \ln (1+\psi(s)) d P(s)-\int_{0}^{t} \psi(s) d s\right),
\end{aligned}
$$

which is (2.39). This ends Example 2.2.

Since (2.39) is equivalent to (2.37), the above argument proves the following result:

Corollary 2.3 Suppose $\psi$ is continuous, $\psi>-1$ and

$$
\int_{0}^{t}(\ln (1+\psi(s)))^{2} d s<\infty .
$$

Then

$$
\exp ^{\diamond}\left(\int_{0}^{t} \psi(s) d Q(s)\right)=\exp \left(\int_{0}^{t} \ln (1+\psi(s)) d P(s)-\int_{0}^{t} \psi(s) d s\right)
$$


Remark The above argument was carried out under the assumption that $\psi \geq$ $-1+\varepsilon$ for some $\varepsilon>0$. However, the identity (2.43) extends to the case (2.42) because

$$
\begin{aligned}
E\left[\left(\int_{0}^{t} \ln (1+\psi(s)) d P(s)\right)^{2}\right] & =E\left[\left(\int_{0}^{t} \ln (1+\psi(s)) d Q(s)+\int_{0}^{t} \ln (1+\psi(s)) d s\right)^{2}\right] \\
& =\int_{0}^{t} \ln (1+\psi(s))^{2} d s+\left(\int_{0}^{t} \ln (1+\psi(s)) d s\right)^{2} .
\end{aligned}
$$

\section{The Mixed Gaussian-Poissonian Case}

Now suppose there are $m$ noise sources, of which $k$ are Gaussian white noises and the remaining $m-k$ are Poissonian. Then we define the combined Gaussian-Poissonian white noise probability measure $\theta$ on $\Omega:=\mathcal{S}^{\prime}\left(\mathbb{R}^{d}\right) \times \cdots \times \mathcal{S}^{\prime}\left(\mathbb{R}^{d}\right)$ ( $m$ factors) by

$$
d \theta\left(\omega_{1}, \ldots, \omega_{m}\right)=d \mu\left(\omega_{1}\right) \cdots d \mu\left(\omega_{k}\right) d \nu\left(\omega_{k+1}\right) \cdots d \nu\left(\omega_{m}\right)
$$

where $\omega=\left(\omega_{1}, \ldots, \omega_{m}\right) \in \Omega$.

For notational simplicity we will from now on assume that $m=2$ and $k=1$. For $\omega=\left(\omega_{1}, \omega_{2}\right) \in \Omega=\mathcal{S}^{\prime}\left(\mathbb{R}^{d}\right) \times \mathcal{S}^{\prime}\left(\mathbb{R}^{d}\right)$ and $\phi=\left(\phi_{1}, \phi_{2}\right) \in \mathcal{S}\left(\mathbb{R}^{d}\right) \times \mathcal{S}\left(\mathbb{R}^{d}\right)$ we define

$$
\langle\omega, \phi\rangle=\left\langle\omega_{1}, \phi_{1}\right\rangle+\left\langle\omega_{2}, \phi_{2}\right\rangle \text {. }
$$

Then by (2.1) we see that

$$
\int_{\Omega} e^{i\langle\omega, \phi\rangle} d \theta(\omega)=\exp \left(-\frac{1}{2}\left\|\phi_{1}\right\|^{2}+\int_{\mathbb{R}^{d}}\left(e^{i \phi_{2}(x)}-1\right) d x\right) .
$$

Let $\Gamma=\mathcal{J} \times \mathcal{J}$ be the set of all pairs $\gamma=(\alpha, \beta)$ of multi-indices $\alpha, \beta \in \mathcal{J}$, and define

$$
M_{\gamma}\left(\omega_{1}, \omega_{2}\right)=H_{\alpha}\left(\omega_{1}\right) G_{\beta}\left(\omega_{2}\right) ; \quad \gamma=(\alpha, \beta) \in \Gamma
$$

For example, this gives (using (2.4a))

$$
\begin{aligned}
& M_{\left(\varepsilon^{(k)}, 0\right)}\left(\omega_{1}, \omega_{2}\right)=H_{\varepsilon^{(k)}}\left(\omega_{1}\right)=\left\langle\omega_{1}, \eta_{k}\right\rangle, \\
& M_{\left(0, \varepsilon^{(\ell)}\right)}\left(\omega_{1}, \omega_{2}\right)=C_{\varepsilon^{(\ell)}}\left(\omega_{2}\right)=\left\langle\omega_{2}, \eta_{\ell}\right\rangle-\bar{\eta}_{\ell} .
\end{aligned}
$$


From tensor product considerations, we see that we have the following analogue of (2.6): Any $f \in L^{2}(\theta)$ can be written (uniquely)

$$
f\left(\omega_{1}, \omega_{2}\right)=\sum_{\gamma \in \Gamma} c_{\gamma} M_{\gamma}\left(\omega_{1}, \omega_{2}\right)
$$

where $c_{\gamma} \in \mathbb{R}$. Moreover, we have the isometry

$$
\|f\|_{L^{2}(\theta)}^{2}=\sum_{\gamma \in \Gamma} c_{\gamma}^{2} \gamma !
$$

where $\gamma !=\alpha ! \beta !$ if $\gamma=(\alpha, \beta) \in \Gamma$.

We proceed as in the list in Section 2. For $\rho \in[-1,1]$ and $q \in \mathbb{Z}$ define the Kondratiev norms

$$
\|F\|_{\rho, q, \theta}^{2}=\sum_{\gamma \in \Gamma} c_{\gamma}^{2}(\gamma !)^{1+\rho}(2 \mathbb{N})^{q \gamma} \text { if } F=\sum_{\gamma \in \Gamma} c_{\gamma} M_{\gamma}(\omega)
$$

where

$$
(2 \mathbb{N})^{q \gamma}=(2 \mathbb{N})^{q \alpha}(2 \mathbb{N})^{q \beta}, \quad \gamma=(\alpha, \beta) \in \Gamma
$$

and the Kondratiev Hilbert spaces

$$
(\mathcal{S})_{\rho, q, \theta}=\left\{F ;\|F\|_{\rho, q, \theta}<\infty\right\}
$$

For $0 \leq \rho \leq 1$ we define the Kondratiev stochastic test function spaces

$$
(\mathcal{S})_{\rho, \theta}=\bigcap_{r \in \mathbb{N}}(\mathcal{S})_{\rho, r, \theta} \quad \text { with projective topology }
$$

and the Kondratiev stochastic distribution spaces

$$
(\mathcal{S})_{-\rho, \theta}=\bigcup_{r \in \mathbb{N}}(\mathcal{S})_{-\rho,-r, \theta} \quad \cdots \text { with inductive topology. }
$$

Furthermore, we define the Hida test function space

$$
(\mathcal{S})_{\theta}:=(\mathcal{S})_{0, \theta}
$$

and the Hida distribution space

$$
(\mathcal{S})_{\theta}^{*}:=(\mathcal{S})_{-0, \theta}
$$


The combined Gaussian-Poissonian white noise $U(x, \omega)$ is defined by

$$
U(x, \omega)=\left(W\left(x, \omega_{1}\right), V\left(x, \omega_{2}\right)\right)
$$

where

$$
W\left(x, \omega_{1}\right)=\sum_{k=1}^{\infty} \eta_{k}(x) H_{\varepsilon^{(k)}}\left(\omega_{1}\right), \ldots . . V\left(x, \omega_{2}\right)=\sum_{\ell=1}^{\infty} \eta_{\ell}(x) C_{\varepsilon^{(\ell)}}\left(\omega_{2}\right)
$$

is Gaussian and Poissonian white noise, respectively. If

$$
F(\omega)=\sum_{\lambda \in \Gamma} a_{\lambda} M_{\lambda}(\omega) \in(\mathcal{S})_{-1, \theta}
$$

and

$$
G(\omega)=\sum_{\delta \in \Gamma} b_{\delta} M_{\delta}(\omega) \in(\mathcal{S})_{-1, \theta}
$$

we define their Wick product $F \diamond G$ by

$$
(F \diamond G)(\omega)=\sum_{\lambda, \delta \in \Gamma} a_{\lambda} b_{\delta} M_{\lambda+\delta}(\omega)=\sum_{\gamma \in \Gamma}\left(\sum_{\lambda+\delta=\gamma} a_{\lambda} b_{\delta}\right) M_{\gamma}(\omega) .
$$

As in the Gaussian case one can prove that

$$
\begin{aligned}
& F, G \in(\mathcal{S})_{-1, \theta} \Rightarrow F \diamond G \in(\mathcal{S})_{-1, \theta}, \\
& f, g \in(\mathcal{S})_{1, \theta} \Rightarrow f \diamond g \in(\mathcal{S})_{1, \theta}
\end{aligned}
$$

\section{Examples}

a) Using (3.5) and (3.6) we get

$$
\begin{aligned}
\left\langle\omega_{1}, \eta_{k}\right\rangle \diamond\left\langle\omega_{2}, \eta_{\ell}\right\rangle & =M_{\left(\varepsilon^{(k)}, 0\right)}(\omega) \diamond\left(M_{\left(0, \varepsilon^{(\ell)}\right)}(\omega)+\bar{\eta}_{\ell}\right) \\
& =M_{\left(\varepsilon^{(k)}, \varepsilon^{(\ell)}\right)}(\omega)+\bar{\eta}_{\ell} M_{\left(\varepsilon^{(k)}, 0\right)}(\omega) \\
& =H_{\varepsilon^{(k)}}\left(\omega_{1}\right) C_{\varepsilon^{(\ell)}}\left(\omega_{2}\right)+\bar{\eta}_{\ell} H_{\varepsilon^{(k)}}\left(\omega_{1}\right) \\
& =\left\langle\omega_{1}, \eta_{k}\right\rangle\left(\left\langle\omega_{2}, \eta_{\ell}\right\rangle-\bar{\eta}_{\ell}\right)+\bar{\eta}_{\ell}\left\langle\omega, \eta_{k}\right\rangle=\left\langle\omega_{1}, \eta_{k}\right\rangle \cdot\left\langle\omega_{2}, \eta_{\ell}\right\rangle .
\end{aligned}
$$

b) The Wick product of Gaussian and Poissonin white noise reads

$$
\begin{aligned}
W\left(x, \omega_{1}\right) \diamond V\left(y, \omega_{2}\right) & =\left(\sum_{k} \eta_{k}(x) H_{\varepsilon^{(k)}}\left(\omega_{1}\right)\right) \diamond\left(\sum_{\ell} \eta_{\ell}(y) C_{\varepsilon^{(\ell)}}\left(\omega_{2}\right)\right) \\
& =\sum_{k, \ell} \eta_{k}(x) \eta_{\ell}(y) H_{\varepsilon^{(k)}}\left(\omega_{1}\right) C_{\varepsilon^{(\ell)}}\left(\omega_{2}\right),
\end{aligned}
$$


which can be written

$$
\sum_{k, \ell} \eta_{k}(x) \eta_{\ell}(y) M_{\left(\varepsilon^{(k)}, \varepsilon^{(\ell)}\right)}(\omega)
$$

and therefore

$$
\begin{aligned}
\|W(x) \diamond V(y)\|_{0,-r, \theta}^{2} & =\sum_{k, \ell} \eta_{k}(x)^{2} \eta_{\ell}(y)^{2} \varepsilon^{(k)} ! \varepsilon^{(\ell)} !(2 \mathbb{N})^{-r\left(\varepsilon^{(k)}, \varepsilon^{(\ell)}\right)} \\
& =\sum_{k, \ell} \eta_{k}(x)^{2} \eta_{\ell}(y)^{2}(2 k)^{-r}(2 \ell)^{-r} \\
& =\left(\sum_{k} \eta_{k}(x)^{2}(2 k)^{-r}\right)\left(\sum_{\ell} \eta_{\ell}(y)^{2}(2 \ell)^{-r}\right)<\infty \quad \text { for } r>1
\end{aligned}
$$

because

$$
\sup _{x, k}\left|\eta_{k}(x)\right|<\infty
$$

Hence

$$
W(x) \diamond V(y) \in(\mathcal{S})_{\theta}^{*} \quad \text { for all } x, y .
$$

c) The preceding example illustrates a general result: If

$$
\begin{aligned}
& F(\omega)=F\left(\omega_{1}\right)=\sum_{\alpha \in \mathcal{J}} a_{\alpha} H_{\alpha}\left(\omega_{1}\right) \in(\mathcal{S})_{-1, \theta} \\
& G(\omega)=G\left(\omega_{2}\right)=\sum_{\beta \in \mathcal{J}} b_{\beta} C_{\beta}\left(\omega_{2}\right) \in(\mathcal{S})_{-1, \theta}
\end{aligned}
$$

then

$$
(F \diamond G)\left(\omega_{1}, \omega_{2}\right)=F\left(\omega_{1}\right) \cdot G\left(\omega_{2}\right)
$$

The proof is an immediate consequence of the definition (3.16):

$$
\begin{aligned}
(F \diamond G)\left(\omega_{1}, \omega_{2}\right) & =\sum_{\alpha, \beta \in \mathcal{J}} a_{\alpha} b_{\beta} M_{(\alpha, \beta)}\left(\omega_{1}, \omega_{2}\right)=\sum_{\alpha, \beta \in \mathcal{J}} a_{\alpha} b_{\beta} H_{\alpha}\left(\omega_{1}\right) C_{\beta}\left(\omega_{2}\right) \\
& =\left(\sum_{\alpha \in \mathcal{J}} a_{\alpha} H_{\alpha}\left(\omega_{1}\right)\right) \cdot\left(\sum_{\beta \in \mathcal{J}} b_{\beta} C_{\beta}\left(\omega_{2}\right)\right)=F\left(\omega_{1}\right) \cdot G\left(\omega_{2}\right) .
\end{aligned}
$$

The Hermite transform of $F(\omega)=\sum_{\gamma \in \Gamma} c_{\gamma} M_{\gamma}(\omega) \in(\mathcal{S})_{-1, \theta}$ is the function $\mathcal{H} F: \mathbb{C}_{0}^{\mathbb{N}} \times$ $\mathbb{C}_{0}^{\mathbb{N}} \rightarrow \mathbb{C}$ defined by

$$
\widetilde{F}(z, w):=\mathcal{H} F(z, w):=\sum_{\gamma \in \Gamma} c_{\gamma} z^{\alpha} w^{\beta} ; \quad \gamma=(\alpha, \beta)
$$


where

$$
z=\left(z_{1}, z_{2}, \ldots\right) \in \mathbb{C}_{0}^{\mathbb{N}}, \quad w=\left(w_{1}, w_{2}, \ldots\right) \in \mathbb{C}_{0}^{\mathbb{N}}
$$

and

$$
z^{\alpha} w^{\beta}=z_{1}^{\alpha_{1}} z_{2}^{\alpha_{2}} \cdots w_{1}^{\beta_{1}} w_{2}^{\beta_{2}} \cdots
$$

if $\alpha=\left(\alpha_{1}, \alpha_{2} \ldots\right), \beta=\left(\beta_{1}, \beta_{2}, \ldots\right)$.

\section{The Hermite transform solution scheme}

Consider a Gaussian-Poissonian white noise differential equation of Wick type in the unknown $F: \mathbb{R}^{d} \rightarrow(\mathcal{S})_{-1, \theta}$ :

$$
A^{\diamond}\left(t, x, \partial_{t}, \nabla_{x}, F, U\right)=0
$$

where $A^{\diamond}$ is the Wick version of a given function $A$. We apply the Hermite transform to this equation and obtain

$$
\widetilde{A}\left(t, x, \partial_{t}, \nabla_{x}, f(z, w), \widetilde{U}(z, w)\right)=0
$$

where $f=\widetilde{F}$. Suppose that for each $z, w$ we can solve the deterministic differential equation (3.25) for $f=f(t, x, z, w)$. Then under certain conditions we get that $f(t, x, z, w)$ is the Hermite transform of some $F: \mathbb{R}^{d} \rightarrow(\mathcal{S})_{-1, \theta}$. Moreover, $F$ solves (3.24). Sufficient conditions for this are given in [6, Theorem 4.1.1] in the Gaussian case and a similar result can easily be proved in the mixed Gaussian-Poissonian case. We omit the details.

\section{Applications to Gaussian-Poissonian SDEs}

We now illustrate the theory by applying it to some stochastic (ordinary and partial) differential equations driven by a Brownian motion and (an independent) Poisson process.

Example 4.1 (Geometric Brownian-Poissonian motion) Consider the equation

$$
d X(t)=a(t) X(t) d t+\phi(t) X(t) d B(t)+\psi(t) X(t) d Q(t)
$$

where $a(t), \phi(t)$, and $\psi(t)$ are deterministic functions, $\psi$ is continuous, $\psi>-1$ and

$$
\int_{0}^{T}\left(|a(t)|+\phi^{2}(t)+(\ln (1+\psi(t)))^{2}\right) d t<\infty .
$$


In view of (2.24) we can rewrite the equation as

$$
\begin{aligned}
\frac{d X(t)}{d t} & =X(t) \diamond[a(t)+\phi(t) W(t)+\psi(t) V(t)] \\
& =X(t) \diamond[a(t)+(\phi(t), \psi(t)) \cdot U(t)] .
\end{aligned}
$$

Applying the usual trick of Wick-multiplying the equation by the integrating factor

$$
\exp ^{\diamond}\left[-\int_{0}^{t}(a(s)+\phi(s) W(s)+\psi(s) V(s)) d s\right]
$$

gives us the solution

$$
X(t)=X(0) \diamond \exp ^{\diamond}\left[\int_{0}^{t} a(s) d s+\int_{0}^{t} \phi(s) d B(s)+\int_{0}^{t} \psi(s) d Q(s)\right] .
$$

If $X(0)$ is constant, this may be written (using (2.18) and Corollary 2.3)

$$
\begin{aligned}
X(t)=X(0) \exp \left[\int_{0}^{t}\left(a(s)-\frac{1}{2} \phi(s)^{2}-\psi(s)\right) d s\right. & \\
& \left.+\int_{0}^{t} \phi(s) d B(s)+\int_{0}^{t} \ln (1+\psi(s)) d P(s)\right] .
\end{aligned}
$$

Example 4.2 Consider the Poisson equation in a Gaussian-Poissonian stochastic medium

$$
\begin{aligned}
\Delta F(x) & =-\phi(s) W(x)-\psi(s) V(x)=-(\phi(x), \psi(x)) \cdot U(x) ; \quad x \in D \\
F(x) & =0 ; \quad x \in \partial D
\end{aligned}
$$

where $\phi(x), \psi(x)$ are bounded, continuous, deterministic functions on a bounded domain $D \subset \mathbb{R}^{d}$ with regular boundary $\partial D$.

To solve this equation we proceed as in the purely Gaussian case (see [6, Section 4.2]): We first take the Hermite transform and obtain the equation

$$
\begin{aligned}
\Delta \widetilde{F}(x, z, w) & =-\phi(s) \widetilde{W}(x, z)-\psi(x) \widetilde{V}(x, w) ; \quad x \in D \\
\widetilde{F}(x, z, w) & =0 ; \quad x \in \partial D
\end{aligned}
$$

with $(z, w) \in \mathbb{C}_{0}^{\mathbb{N}} \times \mathbb{C}_{0}^{\mathbb{N}}$ as parameters. For each fixed $(z, w)$ this equation has the solution

$$
\widetilde{F}(x, z, w)=\int_{D} G(x, y)[\phi(y) \widetilde{W}(y, z)+\psi(y) \widetilde{V}(y, w)] d y
$$


where $G$ is the classical Green's function in $D$. We recognize (4.6) as the Hermite transform of

$$
\begin{array}{r}
F(x)=F(x, \omega)=\int_{D} G(x, y)\left[\phi(y) W\left(y, \omega_{1}\right)+\psi(y) V\left(y, \omega_{2}\right)\right] d y \\
\omega=\left(\omega_{1}, \omega_{2}\right)
\end{array}
$$

This the solution of (4.4), in virtue of the Hermite transform solution scheme. Note that $F(x, \cdot) \in(\mathcal{S})_{\theta}^{*}$ for all $x$, for all dimensions $d$. Moreover, for $d \leq 3$ we actually have $F(x, \cdot) \in L^{2}(\theta)$ because then $G(x, \cdot) \in L^{2}(d y)$ (see [6, p. 5]). And then the solution can be written in the more familiar form

$$
F(x, \omega)=\int_{D} G(x, y)\left[\phi(y) d B\left(y, \omega_{1}\right)+\psi(y) d Q\left(y, \omega_{2}\right)\right] \quad(d \leq 3) .
$$

Similarly the reader can verify that the discussions of the other Wick type stochastic partial differential equations in [6, Chapter 4] carry over to the mixed GaussianPoissonian case.

The Gjessing Formula and the Benth-Gjessing Method in the Mixed Gaussian-Poissonian Case

By inspecting the proof of the Gjessing formula [3] for Gaussian white noise (see, e.g., [6, Theorem 2.10.7], we see that it carries over to the mixed Gaussian-Poissonian case as follows:

Theorem 4.4 (The Gjessing formula, mixed Gaussian-Poissonian case) Suppose $\int_{\mathbb{R}^{d}}\left(\phi(x)^{2}+|\psi(x)|+\ln (1+\psi(x))^{2}\right) d x<\infty$ and $X \in L^{p}(\theta)$ for some $p>1$. Then $X \diamond \exp ^{\diamond}\left(\int_{\mathbb{R}^{d}} \phi d B+\int_{\mathbb{R}^{d}} \psi d Q\right) \in L^{\rho}(\theta)$ for all $\rho<p$ and almost surely we have

$$
\begin{aligned}
\left(X \diamond \operatorname { e x p } ^ { \diamond } \left(\int_{\mathbb{R}^{d}} \phi d B\right.\right. & \left.\left.+\int_{\mathbb{R}^{d}} \psi d Q\right)\right)\left(\omega_{1}, \omega_{2}\right) \\
& =X\left(\omega_{1}-\phi, \omega_{2}-\psi\right) \cdot \exp ^{\diamond}\left(\int_{\mathbb{R}^{d}} \phi d B+\int_{\mathbb{R}^{d}} \psi d Q\right)\left(\omega_{1}, \omega_{2}\right) .
\end{aligned}
$$

In the Gaussian case Benth and Gjessing [2] used the Gjessing formula to solve a class of quasi-linear stochastic differential equations. (See also the exposition in [6, Section 4.8].) This method carries over to the mixed Gaussian-Poissonian case. To illustrate this we discuss the following example: 
Example 4.5 Consider the stochastic differential equation

$$
d X(t)=a(t) X(t)^{\lambda} d t+\phi(t) X(t) d B(t)+\psi(t) X(t) d Q(t)
$$

with $a(t) \geq 0, \phi(t), \psi(t)$ as in Example 4.1, where $\lambda \in \mathbb{R}$ is a constant and we assume $X(0)>0$. The case $\lambda=1$ is covered in Example 4.1 , so we assume $\lambda \neq 1$. The noise interpretation of this equation is (see (2.24))

$$
\frac{d X(t)}{d t}=a(t) X(t)^{\lambda}+X(t) \diamond[\phi(t) W(t)+\psi(t) V(t)]
$$

This equation can be written

$$
\frac{d}{d t}[J(t) \diamond X(t)]=a(t) J(t) \diamond X(t)^{\lambda},
$$

where

$$
J(t)=\exp ^{\diamond}\left(-\int_{0}^{t} \phi(s) W(s) d s-\int_{0}^{t} \psi(s) V(s) d s\right)
$$

Define

$$
Y(t, \omega)=(J(t) \diamond X(t))(\omega)=J(t, \omega) \cdot X\left(t, \omega_{1}+\phi^{(t)}, \omega_{2}+\psi^{(t)}\right)
$$

where $\phi^{(t)}(s)=\mathcal{X}_{[0, t]}(s) \phi(s), \psi^{(t)}(s)=\mathcal{X}_{[0, t]}(s) \psi(s)$. Then by (4.11), (4.9) and (4.13)

$$
\begin{aligned}
\frac{d Y(t, \omega)}{d t} & =a(t)\left(J(t) \diamond X(t)^{\lambda}\right)(\omega) \\
& =a(t)\left(J(t, \omega) \cdot X\left(t, \omega_{1}+\phi^{(t)}, \omega_{2}+\psi^{(t)}\right)^{\lambda}\right) \\
& =a(t) J(t, \omega)\left[J^{-1}(t, \omega) Y(t, \omega)\right]^{\lambda} \\
& =a(t) J(t, \omega)^{1-\lambda} Y(t, \omega)^{\lambda} .
\end{aligned}
$$

For fixed $\omega$ this is a (deterministic) separable differential equation in $Y(t, \omega)$, with solution

$$
Y(t, \omega)=\left[Y(0)^{1-\lambda}+(1-\lambda) \int_{0}^{t} a(s) J(s, \omega)^{1-\lambda} d s\right]^{\frac{1}{1-\lambda}} .
$$

Finally, we use (4.13) to find $X$ :

$$
X\left(t, \omega_{1}, \omega_{2}\right)=J\left(t, \omega_{1}-\phi^{(t)}, \omega_{2}-\psi^{(t)}\right) Y\left(t, \omega_{1}-\phi^{(t)}, \omega_{2}-\psi^{(t)}\right),
$$


where, by (4.12), (2.18) and (2.3),

$$
J\left(t, \omega_{1}, \omega_{2}\right)=\exp \left(-\int_{0}^{t} \phi d B-\int_{0}^{t} \ln (1+\psi) d P-\int_{0}^{t}\left(\frac{1}{2} \phi^{2}-\psi\right) d s\right) .
$$

From (4.15) we conclude that the solution $X(t)$ exists for all $t \geq 0$ if $\lambda<1$ (since $a \geq 0$ ). However, if $\lambda>1$ we may have explosions: $Y(t, \omega)$ only exists up to the first time $t$ such that

$$
X(0)^{1-\lambda}+(1-\lambda) \int_{0}^{t} a(s) J(s, \omega)^{1-\lambda} d s=0 .
$$

For extensions of white noise theory beyond the mixed Gaussian-Poissonian case we refer to [4] and [8].

\section{Acknowledgements}

We wish to thank the Erwin Schrödinger Institute, Vienna and Michael Oberguggenberger for their kind invitation to the Workshop on Nonlinear Theory of Generalized Functions in November/December 1997 and for their hospitality during our stay there.

\section{References}

[1] Benth, F.E., And GJerde, J.: A remark on the equivalence between Poissonian and Gaussian stochastic partial differential equations, Potential Analysis 8 (1998), 179-193.

[2] Benth, F.E., And Gjessing, H.: A non-linear parabolic equation with noise. A reduction method, Manuscripte Nr. 179 (1994), Universität Mannheim.

[3] GJessing, H.: Anticipating Calculus and Wick Products, Dr. Scient. Thesis, University of Bergen, Norway, 1995.

[4] Kondratiev, Y., Streit, L., Westerkamp, W., and Yan, J.: Generalized functions in infinite dimensional analysis, Manuscript, Universität Bielefeld 1995.

[5] Hida, T., Kuo, H.-H., Potthoff, J., And Streit, L.: White Noise Analysis, Kluwer 1993. 
[6] Holden, H., Øksendal, B., Ubøe, J., And Zhang, T.: Stochastic Partial Differential Equations, Birkhäuser 1996.

[7] Oberguggenberger, M., Russo, F.: Nonlinear SPDEs: Colombeau solutions and pathwise limits, In Decreusefond et al. (eds.): Stochastic Analysis and Related Topics VI, Birkhäuser 1998, pp. 319-332.

[8] Us, G.: Towards a coloured noise analysis, Manuscript, Kiev University 1997.

[9] Walsh, J.: An introduction to stochastic partial differential equations, In R. Carmona et al. (eds.): École d'Été de Probabilités de Saint-Flour XIX-1984, Springer Lecture Notes in Mathematics, vol. 1180 (1986), 265-437.

\section{H.HOLDEN}

Department of Mathematical Sciences, Norwegian University of Science and Technology, N-7034 Trondheim, Norway, E-mail: holden@math.ntnu.no

\section{B.ØKSENDAL}

Department of Mathematics, University of Oslo, Box 1053 Blindern, N-0316 Oslo, Norway and Norwegian School of Economics and Business Administration, Helleveien 30, N-5035 Bergen-Sandviken, Norway, E-mail: oksendal@math.uio.no 